\title{
ON ESTIMATION OF THE GAIN OF A DYNAMICAL SYSTEM
}

\section{Bo Wahlberg and Håkan Hjalmarsson}

\author{
Automatic Control Lab and ACCESS \\ School of Electrical Engineering, KTH \\ SE-100 44 Stockholm, Sweden \\ e-mails: bo.wahlberg@ee.kth.se \\ hakan.hjalmarsson@ee.kth.se
}

\author{
Petre Stoica
}

\author{
Department of Information Technology \\ Uppsala University, \\ SE-75 105 Uppsala, Sweden \\ e-mail: ps@it.uu.se
}

\begin{abstract}
We analyze and compare methods to estimate the $\mathscr{L}_{2}$-gain ( $\mathscr{H}_{\infty}$-norm) of a stable linear dynamical system, that is the maximum of the absolute value of the corresponding frequency response. The standard approach is to estimate a parametric model of the system, which then is used for gain calculation. An asymptotic error variance expression for $\mathscr{H}_{\infty}$-norm estimates based on finite impulse response (FIR) models is presented. We then study the problem of finding the minimum variance excitation signal that satisfies a given error variance bound for the FIR model gain estimate. It is shown that a sinusoidal signal with frequency equal to the peak frequency of the system is minimum variance optimal. The second approach to gain estimation is based on iterative experiments, and is inspired by numerical methods eigenvalue calculation. The asymptotic statistical properties of such gain estimation methods are compared to the FIR model approach. A transparent expression for the additional variance cost of using iterative experiments is derived. Finally, we present some ideas for input sequence estimation based on iterative experiments.
\end{abstract}

\section{INTRODUCTION}

Consider a scalar discrete time asymptotically stable timeinvariant linear dynamical system with impulse response sequence $\left\{g_{k}\right\}$, input signal sequence $\{u(t)\}$, output signal sequence $\{y(t)\}$, and additive zero mean white noise $\{e(t)\}$ with variance $\sigma_{e}^{2}$. The input to output relation is given by

$$
y(t)=\sum_{k=0}^{\infty} g_{k} u(t-k)+e(t) .
$$

The corresponding frequency response function is defined by

$$
G\left(e^{i \omega}\right)=\sum_{k=0}^{\infty} g_{k} e^{-i \omega k}, \quad \omega \in(-\pi, \pi], \quad i=\sqrt{-1},
$$

and is a most important tool in feedback control design. Frequency response function estimation is a fundamental prob-

This work was partially supported by the Swedish Research Council and the Linnaeus Center ACCESS at KTH. lem in both spectral estimation and system identification, see e.g. [16] and [9].

The $\mathscr{L}_{2}$-norm of a sequence $\{x(t), t=1,2, \ldots\}$ is defined by $\|x\|_{2}=\sqrt{\sum_{t=1}^{\infty} x^{2}(t)}$. We will study the $\mathscr{L}_{2}$-gain $\beta$ of a dynamical system:

$$
\beta=\sup _{u \neq 0,\|u\|_{2}^{2} \leq \gamma} \frac{\|y\|_{2}}{\|u\|_{2}}
$$

that is the largest $\mathscr{L}_{2}$-norm amplification from input signal $u(t)$ to output signal $y(t)$. For asymptotically stable linear systems the $\mathscr{L}_{2}$-gain equals the $\mathscr{H}_{\infty}$-norm, that is the maximum of the absolute value of the frequency response, $\beta=$ $\max _{\omega}\left|G\left(e^{i \omega}\right)\right|$. The maximizing input is a sinusoidal signal with corresponding peak frequency, see, e.g. [6]. For linear systems the gain (2) does not depend on the choice of $\gamma$.

The small gain theorem, see, e.g. [7], is a most useful tool for validating the stability of a feedback system. It requires the knowledge of an upper bound of the $\mathscr{L}_{2}$-gain of the corresponding open loop system. This motivates deriving accurate and reliable gain estimation methods. A common approach is to use a System Identification (SI) algorithm, see [9], [12] or [14], and then calculate the $\mathscr{L}_{2}$-gain of the identified model. We will first study gain-estimation based on Finite Impulse Response (FIR) models, and present the corresponding asymptotic gain error variance expression. If it is possible to choose the excitation in the data collection experiment, an interesting problem is to find the input signal that minimizes the uncertainty of the estimate. This makes sense only if we constrain for example the power of the input signal as otherwise the error can be made arbitrarily small. The problem of finding optimal input signals for parameter estimation in dynamic systems is a classical topic. An excellent survey of the field up to 1974 is given in [11]. There has recently been a renewed interest in the optimal input design problem, and a survey of the current state of the art is given in [5].

A fundamental problem with optimal input design is that the solution in general depends on the unknown true system. An approach, recently studied in [17], is non-parametric gain estimation methods based on input signal optimization and iterative experiments. The basic idea is to let the system it- 
self generate the optimal input signal for gain estimation. No explicit model of the system is required. An introduction to this approach will be presented together with a variance expression for the corresponding gain estimate. The objective of this paper is to compare this expression with the FIR response result. This gives insights into the cost of using iterative experiments.

The outline of this paper is as follows. Section 2 contains a variance analysis of gain estimates based on FIR models, and the optimal solution to the minimum variance input design problem is derived. Section 3 concerns gain estimation using iterative experiments. In this section the relation to the FIR approach is studied in detail. Section 4 is about input estimation for the gain problem, while Section 5 concludes the paper.

\section{FIR MODEL GAIN ESTIMATION}

We will consider identification of $n$ :th order finite impulse response (FIR) systems, for which $g_{k}=0, k>n$. Furthermore, it will be assumed that $u(t)=0$ for $t \leq 0$ and $t>N$, i.e., zero initial and final conditions. The input to output relation (1) for a given input sequence $\{u(t), t=1, \ldots, N\}$ can then be written in matrix notation as

$$
\begin{aligned}
& \mathbf{y}=\mathbf{U g}+\mathbf{e}, \\
& \mathbf{y}=[y(1), y(2), \ldots, y(N+n)]^{T}, \\
& \mathbf{g}=\left[g_{0}, g_{1}, \ldots, g_{n}\right]^{T}, \mathbf{e}=[e(1), e(2), \ldots, e(N+n)]^{T},
\end{aligned}
$$

and $\mathbf{U}$ is a $n+N$ times $n+1$ lower triangular Toeplitz matrix with first column $\mathbf{u}=[u(1), u(2), \ldots u(N), 0, \ldots, 0]^{T}$. If the zero initial or the zero final conditions are not satisfied, the first $n$ or the last $n$ rows of $\mathbf{U}$ and $\mathbf{y}$ should be removed for (3) to hold.

Given observations $\mathbf{u}$ and $\mathbf{y}$, the least squares estimate of the impulse response vector $\mathbf{g}$ is

$$
\hat{\mathbf{g}}=\left[\frac{1}{N} \mathbf{U}^{T} \mathbf{U}\right]^{-1}\left[\frac{1}{N} \mathbf{U}^{T} \mathbf{y}\right] .
$$

Here we estimate $n+1$ parameters from at best $N+n$ observations. Typically, for $N$ observations the number of parameters, $n+1$, needs to be at most on the order of $\sqrt{N / \log N}$ for almost sure convergence, see [10].

\subsection{Variance Analysis}

The statistical properties of $\hat{\mathbf{g}}$ are well known, see e.g., [9]. We will use $\mathbf{g}_{o}$ to denote the true system parameters, and $\Delta \mathbf{g}=\hat{\mathbf{g}}-$ $\mathbf{g}_{o}$ for the corresponding the estimation error. The covariance matrix of the least squares estimate error equals

$$
\operatorname{Cov}\{\hat{\mathbf{g}}\}=\mathrm{E}\left\{\Delta \mathbf{g} \Delta \mathbf{g}^{T}\right\}=\mathbf{P}_{N}, \quad \mathbf{P}_{N}=\frac{\sigma_{e}^{2}}{N}\left[\frac{1}{N} \mathbf{U}^{T} \mathbf{U}\right]^{-1} .
$$

The asymptotic (large $N$ ) error covariance matrix of the least squares estimate (4) is

$$
\begin{aligned}
\operatorname{AsCov}\{\hat{\mathbf{g}}\} & =\mathbf{P}, \quad \mathbf{P}=\frac{\sigma_{e}^{2}}{N} \mathbf{R}^{-1}, \\
\mathbf{R}_{j k} & =r_{\tau}=\overline{\mathrm{E}}\{u(t) u(t-\tau)\}, \quad \tau=|j-k|,
\end{aligned}
$$

where $\bar{E}$ denotes quasi-stationary expectation, defined in [9]. For a stationary zero mean stochastic process this equals ordinary expectation and $r_{\tau}=\mathrm{E}\{u(t) u(t-\tau)\}$ is the covariance function. We use As to denote variance results that are asymptotic in the number of data points, $N$.

Consider the estimated frequency response, the true frequency response and the corresponding estimation error

$$
\begin{aligned}
\hat{G}\left(e^{i \omega}\right) & =\Gamma^{*}\left(e^{i \omega}\right) \hat{\mathbf{g}}, \quad \Gamma\left(e^{i \omega}\right)=\left[1, e^{i \omega}, \ldots, e^{i n \omega}\right]^{T} \\
G_{o}\left(e^{i \omega}\right) & =\Gamma^{*}\left(e^{i \omega}\right) \mathbf{g}_{o} \\
\Delta G\left(e^{i \omega}\right) & =\hat{G}\left(e^{i \omega}\right)-G_{o}\left(e^{i \omega}\right)=\Gamma^{*}\left(e^{i \omega}\right) \Delta \mathbf{g}
\end{aligned}
$$

where super-index $*$ denotes conjugate transpose. Using $\left|G\left(e^{i \omega}\right)\right|^{2}=G\left(e^{i \omega}\right) G^{*}\left(e^{i \omega}\right)$, a first order perturbation analysis gives

$$
\begin{aligned}
\Delta\left|G\left(e^{i \omega}\right)\right| & \approx \frac{1}{2\left|G_{o}\left(e^{i \omega}\right)\right|} \Delta\left|G\left(e^{i \omega}\right)\right|^{2}, \\
\Delta\left|G\left(e^{i \omega}\right)\right|^{2} & \approx \Delta G\left(e^{i \omega}\right) G_{o}^{*}\left(e^{i \omega}\right)+G_{o}\left(e^{i \omega}\right) \Delta G^{*}\left(e^{i \omega}\right) .
\end{aligned}
$$

Combining this with (10) gives ${ }^{1}$

$$
\Delta\left|G\left(e^{i \omega}\right)\right| \approx \frac{1}{\left|G_{o}\left(e^{i \omega}\right)\right|}\left[\operatorname{Re}\left\{G_{o}\left(e^{i \omega}\right) \Gamma\left(e^{i \omega}\right)\right\}\right]^{T} \Delta \mathbf{g},
$$

and thus

$$
\begin{aligned}
& \operatorname{As} \operatorname{Var}\left\{\left|\hat{G}\left(e^{i \omega}\right)\right|\right\}= \\
& \frac{1}{\left|G_{o}\left(e^{i \omega}\right)\right|^{2}}\left[\operatorname{Re}\left\{G_{o}\left(e^{i \omega}\right) \Gamma\left(e^{i \omega}\right)\right\}\right]^{T} \mathbf{P}\left[\operatorname{Re}\left\{G_{o}\left(e^{i \omega}\right) \Gamma\left(e^{i \omega}\right)\right\}\right] .
\end{aligned}
$$

Define

$$
\begin{aligned}
\omega_{p}^{o}=\arg \max _{\omega}\left|G_{o}\left(e^{i \omega}\right)\right|, & \beta_{o}=\left|G_{o}\left(e^{i \omega_{p}^{o}}\right)\right| \\
\hat{\omega}_{p}=\arg \max _{\omega}\left|\hat{G}\left(e^{i \omega}\right)\right|, & \hat{\beta}=\left|\hat{G}\left(e^{i \hat{\omega}_{p}}\right)\right| \\
\Delta \omega_{p}=\hat{\omega}_{p}-\omega_{p}^{o}, & \Delta \beta=\hat{\beta}-\beta_{o} .
\end{aligned}
$$

The parameter $\beta_{o}$ is the $\mathscr{H}_{\infty}$-norm of $G_{o}\left(e^{i \omega}\right)$, i.e.

$$
\beta_{o}=\left|G_{o}\left(e^{i \omega_{p}^{o}}\right)\right| \geq\left|G_{o}\left(e^{i \omega}\right)\right|, \quad \forall \omega,
$$

and $\hat{\beta}$ is a corresponding estimate based on $\hat{G}\left(e^{i \omega}\right)$.

\footnotetext{
${ }^{1} \operatorname{Re}\{z\}$ denotes the real part and $\operatorname{Im}\{z\}$ denotes the imaginary part of a complex number $z$.
} 
Proposition 1 The asymptotic error variance of the least squares based estimate of the $\mathscr{H}_{\infty}$-norm of $G_{o}\left(e^{i \omega}\right)$, defined by (15), equals

$$
\begin{aligned}
& \operatorname{As} \operatorname{Var}\{\hat{\beta}\}= \\
& \frac{1}{\left|G_{o}\left(e^{i \omega_{p}^{o}}\right)\right|^{2}}\left[\operatorname{Re}\left\{G_{o}\left(e^{i \omega_{p}^{o}}\right) \Gamma\left(e^{i \omega_{p}^{o}}\right)\right\}\right]^{T} \mathbf{P}\left[\operatorname{Re}\left\{G_{o}\left(e^{i \omega_{p}^{o}}\right) \Gamma\left(e^{i \omega_{p}^{o}}\right)\right\}\right] .
\end{aligned}
$$

Proof: A first order perturbation analysis gives

$$
\Delta \beta \approx\left[\left.\frac{\partial\left|G_{o}\left(e^{i \omega}\right)\right|}{\partial \omega}\right|_{\omega=\omega_{p}^{o}}\right] \Delta \omega_{p}+\left[\left.\frac{\partial\left|G\left(e^{i \omega_{p}^{o}}\right)\right|}{\partial \mathbf{g}}\right|_{\mathbf{g}=\mathbf{g}_{0}}\right]^{T} \Delta \mathbf{g},
$$

where the first term of the right hand side is zero since $\omega_{p}^{o}$ is a critical point (maximum). The variance of the second term is $\operatorname{As} \operatorname{Var}\left\{\left|\hat{G}\left(e^{i \omega_{p}^{o}}\right)\right|\right\}$, which is given by (14). Q.E.D

This result implies that there is no asymptotic variance cost due to uncertainty in frequency since the corresponding derivative is zero at $\omega_{p}^{o}$.

\subsection{Optimal Input Design}

Consider the optimal input design problem

$$
\mathrm{P} 1: \quad \min _{\mathbf{r}} r_{0} \quad \text { s.t. } \quad \mathbf{c}^{T} \mathbf{R}^{-1} \mathbf{c} \leq \frac{N \varepsilon}{\sigma_{e}^{2}}, \quad \mathbf{R} \geq 0,
$$

where

$$
\begin{aligned}
& \mathbf{c}=\frac{1}{\left|G_{o}\left(e^{i \omega_{p}^{o}}\right)\right|}\left[\operatorname{Re}\left\{G_{o}\left(e^{i \omega_{p}^{o}}\right) \Gamma\left(e^{i \omega_{p}^{o}}\right)\right\}\right], \\
& \mathbf{r}=\left[r_{0}, r_{1}, \ldots, r_{n}\right]^{T}, \quad r_{\tau}=\overline{\mathrm{E}}\{u(t) u(t-\tau)\},
\end{aligned}
$$

and $\mathbf{R}$ is the symmetric Toeplitz matrix with $\mathbf{r}$ as the first column, see (7). This problem corresponds to minimizing the variance of the input signal $r_{0}=\overline{\mathrm{E}}\left\{u^{2}(t)\right\}$ subject to the quality constraint $A s \operatorname{Var}\{\hat{\beta}\} \leq \varepsilon$. Using Schur complements it is possible to show, see [13], that Problem P1 is equivalent to the optimization problem

$$
\text { P2: } \min _{\mathbf{r}} r_{0} \quad \text { s.t. } \quad \mathbf{R} \geq \frac{\sigma_{e}^{2}}{N \varepsilon} \mathbf{c c}^{T} .
$$

We would like to compare the optimal solution of (18) to the case when a sinusoidal signal $u(t)=C \cos (\bar{\omega} t)$ is used. The corresponding quasi-stationary covariance function $r_{k}$ of the latter input is given by

$$
r_{0}=\left\{\begin{array}{ll}
C^{2}, & \bar{\omega}=0, \pi \\
\frac{C^{2}}{2}, & \text { otherwise }
\end{array}, \quad r_{k}=r_{0} \cos (\bar{\omega} k), \quad k \geq 1,\right.
$$

and thus

$$
\mathbf{R}=r_{0} \operatorname{Re}\left\{\Gamma\left(e^{i \bar{\omega}}\right) \Gamma^{*}\left(e^{i \bar{\omega}}\right)\right\}
$$

For $n \geq 1$ the rank of the matrix $\mathbf{R}$ is 1 for $\bar{\omega}=0, \pi$ and otherwise 2 . Therefore it is only possible to estimate functions of the frequency response $G\left(e^{i \bar{\omega}}\right)$ at $\omega=\bar{\omega}$, and we need to use a pseudo-inverse of $\mathbf{R}$ in the frequency response variance expression (14),

$$
\begin{aligned}
& \operatorname{As} \operatorname{Var}\left\{\left|\hat{G}\left(e^{i \omega}\right)\right|\right\}=\frac{\sigma_{e}^{2}}{N} \mathbf{c}^{T} \mathbf{R}^{\dagger} \mathbf{c}= \\
& \frac{\sigma_{e}^{2}}{N\left|G_{o}\left(e^{i \omega}\right)\right|^{2} r_{0}} \mathbf{g}_{o}^{T}\left[\operatorname{Re}\left\{\Gamma\left(e^{i \omega}\right) \Gamma^{*}\left(e^{i \omega}\right)\right\}\right]\left[\operatorname{Re}\left\{\Gamma\left(e^{i \omega}\right) \Gamma^{*}\left(e^{i \omega}\right)\right\}\right]^{\dagger} \\
& \times\left[\operatorname{Re}\left\{\Gamma\left(e^{i \omega}\right) \Gamma^{*}\left(e^{i \omega}\right)\right\}\right] \mathbf{g}_{o} \\
& =\frac{\sigma_{e}^{2}}{N\left|G_{o}\left(e^{i \omega}\right)\right|^{2} r_{0}} \mathbf{g}_{o}^{T}\left[\operatorname{Re}\left\{\Gamma\left(e^{i \omega}\right) \Gamma^{*}\left(e^{i \omega}\right)\right\}\right] \mathbf{g}_{o}=\frac{\sigma_{e}^{2}}{N r_{0}}
\end{aligned}
$$

Here we have several times used the fact that $G_{o}\left(e^{i \omega}\right)=$ $\Gamma^{*}\left(e^{i \omega}\right) \mathbf{g}_{o}$.

We will now show that a sinusoidal signal with frequency $\omega_{p}^{o}$ is optimal for estimating the $\mathscr{H}_{\infty}$-norm of a FIR system, see (15). The practical problem is, of course, that we do not know the peak frequency $\omega_{p}^{o}$ in advance. Proposition 1 shows that the solution is robust to frequency errors.

Proposition 2 The optimal solution of $P 1$ and $P 2$ is a sinusoidal signal with frequency $\omega_{p}^{o}$ and power $r_{0}=\sigma_{e}^{2} /(N \varepsilon)$.

Proof: Since $G_{o}\left(e^{i \omega}\right)=\Gamma^{*}\left(e^{i \omega}\right) \mathbf{g}_{o}$, we have

$$
\mathbf{g}_{o}^{T} \mathbf{c}=\operatorname{Re}\left\{\left|G_{o}\left(e^{i \omega_{p}^{o}}\right)\right|\right\}=\left|G_{o}\left(e^{i \omega_{p}^{o}}\right)\right|
$$

The constraint in $\mathrm{P} 2$ implies that

$$
\mathbf{g}_{o}^{T} \mathbf{R} \mathbf{g}_{o} \geq \frac{\sigma_{e}^{2}}{N \varepsilon} \mathbf{g}_{o}^{T} \mathbf{c c}^{T} \mathbf{g}_{o}=\frac{\sigma_{e}^{2}}{N \varepsilon}\left|G_{o}\left(e^{i \omega_{p}^{o}}\right)\right|^{2} .
$$

Using the definition of $\omega_{p}^{o},(16)$ :

$$
\begin{aligned}
& \mathbf{g}_{o}^{T} \mathbf{R g}_{o}=\frac{1}{2 \pi} \int_{-\pi}^{\pi}\left|G_{o}\left(e^{i \omega}\right)\right|^{2} \Phi_{u}(\omega) d \omega \\
& \leq\left|G_{o}\left(e^{i \omega_{p}^{o}}\right)\right|^{2} \frac{1}{2 \pi} \int_{-\pi}^{\pi} \Phi_{u}(\omega) d \omega=\left|G_{o}\left(e^{i \omega_{p}^{o}}\right)\right|^{2} r_{0}
\end{aligned}
$$

Hence a necessary condition for optimality is $r_{0} \geq \sigma_{e}^{2} /(N \varepsilon)$, but according to (24) a sinusoidal signal with frequency $\omega_{p}^{o}$ and power $r_{0}=\sigma_{e}^{2} /(N \varepsilon)$ satisfies the constraints and is thus an optimal solution. Q.E.D.

Remark: Proposition 2 and the result (24) show that the noise to input ratio

$$
\frac{\sigma_{e}^{2}}{N r_{0}}
$$

is a fundamental lower bound on $\operatorname{As} \operatorname{Var}\left\{\left|\hat{G}\left(e^{i \omega_{p}^{o}}\right)\right|\right\}$ for any input excitation signal $u(t)$ with variance $r_{0}$. This result is independent of the FIR model order. This lower bound is achieved when using a sinusoidal signal with frequency equal 
to the peak frequency. As shown in [18], this result is in general not true at other frequencies. For example a sinusoidal signal with frequency $\bar{\omega}$ is minimum variance optimal for estimating the frequency response for a first order FIR response model at frequency $\omega$, if $\bar{\omega}$ and $\omega$ are related as:

$$
\cos (\bar{\omega})=\frac{\cos (\omega)}{1+|\sin (\omega)|} .
$$

For a first order FIR system the peak frequency is $\omega_{p}^{o}=0$ or $\pi$ and Proposition 2 will hold.

Remark: As mentioned above it is not possible to estimate the full impulse response vector of FIR systems of order larger than two with a sinusoidal input signal with frequency $\omega$. However,

$$
\hat{G}\left(e^{i \omega}\right)=\Gamma^{*}\left(e^{i \omega}\right)\left[\frac{1}{N} \mathbf{U}^{T} \mathbf{U}\right]^{\dagger}\left[\frac{1}{N} \mathbf{U}^{T} \mathbf{y}\right],
$$

where we use the pseudo-inverse, is a valid estimate. A more efficient implementation for $\omega_{p}^{o} \neq 0, \pi$ is to estimate the frequency response from a first order FIR model, i.e., with two free parameters. For a sinusoidal input signal with frequency $\omega$ the two frequency response estimates will have the same asymptotic properties.

\section{GAIN ESTIMATION USING ITERATIVE EXPERIMENTS}

The input to output relation (1) with zero initial conditions and input sequence $\{u(t), t=1, \ldots, N\}$ can be written in matrix notation as

$$
\mathbf{y}=\mathbf{G u}+\mathbf{e},
$$

where matrix $\mathbf{G}$ is an $N$ times $N$ lower triangular Toeplitz matrix with first column $\left[g_{0}, g_{1}, \ldots, g_{N-1}, 0, \ldots, 0\right]^{T}$. This is just another way to write (3). Here we do not include $y(t)$, $t=N+1, \ldots, N+n$, but it is easy to modify the analysis to include such measurements. Notice that we do not assume a FIR system.

To start with assume that there is no noise $e(t)$. The gain cost function

$$
V(\mathbf{u})=\frac{\mathbf{y}^{T} \mathbf{y}}{\mathbf{u}^{T} \mathbf{u}}=\frac{\mathbf{u}^{T} \mathbf{G}^{T} \mathbf{G u}}{\mathbf{u}^{T} \mathbf{u}}, \quad \mathbf{u} \neq 0
$$

will be maximized with respect to the finite sequence $\mathbf{u}$ in order to find a lower bound on the $\mathscr{L}_{2}$-gain. The function (27) is called the Rayleigh quotient in numerical analysis, see [4]. It is well known that $V(\mathbf{u}) \leq \lambda^{\max }$ (the maximum eigenvalue of the matrix $\mathbf{G}^{T} \mathbf{G}$ ) with equality if $\mathbf{u}=\overline{\mathbf{u}}$, a corresponding "maximum" eigenvector. By letting the length of the sequence u go to infinity, the $\mathscr{L}_{2}$-gain equals $\beta=\lim _{N \rightarrow \infty} \sqrt{\lambda \max }$. The Toeplitz structure of $\mathbf{G}$ and the stability assumption imply that $\lambda^{\max }$ tends to $\max _{\omega}\left|\sum_{k=0}^{\infty} g_{k} e^{-i \omega k}\right|^{2}$ as $N \rightarrow \infty$.

As discussed in the previous section we can estimate the impulse response using a FIR model approximation to find the matrix $\mathbf{G}^{T} \mathbf{G}$ and numerically solve the corresponding maximum eigenvalue problem. A problem with the model estimation approach is that we have to set $g_{k}=0$ for $k \leq n$, where $n \ll N$, for the FIR estimates to converge. However, in order to use an iterative algorithm for eigenvalue calculations it is enough evaluate $\mathbf{G}^{T} \mathbf{G u}=\mathbf{G}^{T} \mathbf{y}$ for a given $\mathbf{u}$. For a linear time-invariant system (1) this can be done as follows: Perform a first experiment with input $\mathbf{u}$ and corresponding output $\mathbf{y}$. In a second experiment apply the reversed time output sequence $\tilde{\mathbf{y}}=[y(N), y(N-1), \ldots, y(1)]^{T}$ as input to the system. Let the corresponding output sequence be $[z(1), z(2), \ldots, z(N)]^{T}$ and denote its time reversed version by $\tilde{\mathbf{z}}=[z(N), z(N-1), \ldots, z(1)]^{T}$. Due to the Toeplitz structure,

$$
\tilde{\mathbf{z}}=\mathbf{G}^{T} \mathbf{y}=\mathbf{G}^{T} \mathbf{G u} .
$$

This means that $\mathbf{G}^{T} \mathbf{G u}$, and $V(\mathbf{u})$, can be determined from two experiments without explicitly knowing the matrix $\mathbf{G}$.

It is possible to use the same trick for the frequency response. Let $u(t)=\cos (\omega t)$ and assume that there is no transients. Then

$$
\begin{aligned}
& y(t)=\left|G\left(e^{i \omega}\right)\right| \cos \left(\omega t+\arg \left\{G\left(e^{i \omega}\right)\right\}\right), \\
& z(t)=\left|G\left(e^{i \omega}\right)\right|^{2} \cos \left(-\omega t+\arg \left\{G\left(e^{i \omega}\right)\right\}+\arg \left\{G\left(e^{-i \omega}\right)\right\}\right), \\
& \tilde{z}(t)=\left|G\left(e^{i \omega}\right)\right|^{2} \cos (\omega t)
\end{aligned}
$$

since $\arg \left\{G\left(e^{i \omega}\right)\right\}+\arg \left\{G\left(e^{-i \omega}\right)\right\}=0$. The input signal to the second experiment will have a power proportional to $\left|G\left(e^{i \omega}\right)\right|^{2}$ and should be scaled if there are power limitations.

The noise corrupted version of (28) is

$$
\tilde{\mathbf{z}}=\mathbf{G}^{T} \mathbf{G u}+\mathbf{G}^{T} \mathbf{e}_{1}+\tilde{\mathbf{e}}_{2},
$$

where $\mathbf{e}_{1}$ is the noise vector from the first experiment and $\tilde{\mathbf{e}}_{2}$ originates from the second experiment (with $\tilde{\mathbf{e}}_{2}$ denoting the reversed time version of $\mathbf{e}_{2}$ ).

\subsection{Statistical Analysis of Eigenvalue Estimate}

Assume that a maximum eigenvector $\overline{\mathbf{u}}$ of $\mathbf{G}^{T} \mathbf{G}$ is known, $\mathbf{G}^{T} \mathbf{G} \overline{\mathbf{u}}=\lambda^{\max } \overline{\mathbf{u}}$, and that the corresponding sequence is used as input to the system. Then (30) becomes

$$
\tilde{\mathbf{z}}=\lambda^{\max } \overline{\mathbf{u}}+\mathbf{G}^{T} \mathbf{e}_{1}+\tilde{\mathbf{e}}_{2},
$$

where the noise contribution in (31) has covariance matrix $\sigma_{e}^{2}\left(\mathbf{G}^{T} \mathbf{G}+\mathbf{I}\right)$. The best linear unbiased estimate of $\lambda^{\max }$ is given by the weighted Least Squares (LS) problem

$$
\min _{\lambda}[\tilde{\mathbf{z}}-\lambda \overline{\mathbf{u}}]^{T}\left(\mathbf{G}^{T} \mathbf{G}+\mathbf{I}\right)^{-1}[\tilde{\mathbf{z}}-\lambda \overline{\mathbf{u}}] .
$$

The weighting matrix is unknown, but since $\overline{\mathbf{u}}$ is an eigenvector of $\mathbf{G}^{T} \mathbf{G}$ we have $\left(\mathbf{G}^{T} \mathbf{G}+\mathbf{I}\right)^{-1} \overline{\mathbf{u}}=1 /\left(1+\lambda^{\max }\right) \overline{\mathbf{u}}$. From this it follows that the minimizing solution to (32) equals the standard (without weighting) LS solution

$$
\hat{\lambda}^{\max }=\frac{\overline{\mathbf{u}}^{T} \tilde{\mathbf{z}}}{\overline{\mathbf{u}}^{T}} .
$$


Substituting expression (31) in (33) gives

$$
\hat{\lambda}^{\max }=\lambda^{\max }+\frac{\overline{\mathbf{u}}^{T}\left(\mathbf{G}^{T} \mathbf{e}_{1}+\tilde{\mathbf{e}}_{2}\right)}{\overline{\mathbf{u}}^{T} \overline{\mathbf{u}}} .
$$

The error term due to noise has zero mean value and variance

$$
\mathrm{E}\left\{\left(\frac{\overline{\mathbf{u}}^{T}\left(\mathbf{G}^{T} \mathbf{e}_{1}+\tilde{\mathbf{e}}_{2}\right)}{\overline{\mathbf{u}}^{T} \overline{\mathbf{u}}}\right)^{2}\right\}=\frac{\sigma_{e}^{2}\left(\lambda^{\max }+1\right)}{\|\overline{\mathbf{u}}\|_{2}^{2}},
$$

with $\|\overline{\mathbf{u}}\|_{2}^{2}=\overline{\mathbf{u}}^{T} \overline{\mathbf{u}}$. Here we have exploited the fact that $\{e(t)\}$ is white noise and again that $\overline{\mathbf{u}}$ is an eigenvector of $\mathbf{G}^{T} \mathbf{G}$. Variance expression (34) can be viewed as a lower bound on the variance of methods that also estimate $\mathbf{u}$. It nicely captures how the error depends on energy of the input signal $\|\overline{\mathbf{u}}\|_{2}^{2}$, the gain $\lambda^{\max }$ and the noise variance $\sigma_{e}^{2}$.

To find $\tilde{\mathbf{z}}$ in the second experiment we have applied the reversed output $\tilde{\mathbf{y}}$ as input sequence. The variance of $\{\tilde{y}(t)\}$ equals

$$
\lambda^{\max } r_{0}+\sigma_{e}^{2} .
$$

If we scale $\tilde{\mathbf{y}}$ to be of the same size as $\overline{\mathbf{u}}$, i.e., use

$$
\mathbf{y}_{2}=K \tilde{\mathbf{y}}, \quad \frac{1}{K^{2}}=\lambda^{\max }+\frac{\sigma_{e}^{2}}{r_{0}},
$$

as the input to the second experiment, we obtain

$$
\hat{\lambda}^{\max }=\lambda^{\max }+\frac{\overline{\mathbf{u}}^{T}\left(\mathbf{G}^{T} \mathbf{e}_{1}+\tilde{\mathbf{e}}_{2} / K\right)}{\overline{\mathbf{u}}^{T} \overline{\mathbf{u}}} .
$$

and

$$
\begin{aligned}
\mathrm{E}\left\{\left(\hat{\lambda}^{\max }-\lambda^{\max }\right)^{2}\right\} & =\frac{\sigma_{e}^{2}\left(\lambda^{\max }+1 / K^{2}\right)}{\|\overline{\mathbf{u}}\|_{2}^{2}} \\
& =\frac{\sigma_{e}^{2}\left(2 \lambda^{\max }+\sigma_{e}^{2} / r_{0}\right)}{\|\overline{\mathbf{u}}\|_{2}^{2}} .
\end{aligned}
$$

Observe that $\|\overline{\mathbf{u}}\|_{2}^{2}$ typically is of order $N$. This means that $\hat{\lambda}^{\max }$ is a consistent estimate of the gain as $N$ tends to infinity.

The corresponding result can also be obtained from the frequency response expression

$$
\tilde{z}(t)=\left|G\left(e^{i \omega}\right)\right|^{2} \cos (\omega t)+\mathbf{v}_{1}+\frac{\tilde{\mathbf{e}}_{2}}{K},
$$

where the spectral density corresponding to $\mathbf{v}_{1}=\mathbf{G}^{T} \mathbf{e}_{1}$ is $\sigma_{e}^{2}\left|G\left(e^{i \omega}\right)\right|^{2}$ and $\left\{\tilde{\mathbf{e}}_{2}\right\}$ is white noise with variance $\sigma_{e}^{2}$. The noise terms $\left\{\mathbf{v}_{1}\right\}$ and $\left\{\tilde{\mathbf{e}}_{2}\right\}$ are uncorrelated. The least squares estimate of $\left|G\left(e^{i \omega}\right)\right|^{2}$ equals

$$
\left|\hat{G}\left(e^{i \omega}\right)\right|^{2}=\frac{\sum_{t=1}^{N} \tilde{z}(t) \cos (\omega t)}{\sum_{t=1}^{N} \cos ^{2}(\omega t)},
$$

with asymptotic variance, $c . f$. [15],

$$
\frac{\Phi_{v}(\omega)}{N r_{0}}
$$

where $\Phi_{v}(\omega)$ is the power spectral density of the noise $\mathbf{v}_{1}+$ $\tilde{\mathbf{e}}_{2} / K$ evaluated at the frequency of the input signal. Here

$$
\Phi_{v}(\omega)=\sigma_{e}^{2}\left|G\left(e^{i \omega}\right)\right|^{2}+\sigma_{e}^{2} / K^{2},
$$

which at $\omega=\omega_{p}^{o}$ and with $\lambda^{\max }=\left|G\left(e^{i \omega_{p}^{o}}\right)\right|^{2}$ is equivalent to expression (35).

\subsection{Accuracy Comparison}

In this section we compare the asymptotic accuracy of the direct approach of estimating the $\mathscr{H}_{\infty}$-norm with that of the iterative experiment approach. First note that, see [2],

$$
\left|G\left(e^{i \omega_{p}^{o}}\right)\right| \approx \sqrt{\lambda \max }
$$

for large $N$, and thus

$$
\operatorname{As} \operatorname{Var}\left\{\left|\hat{G}\left(e^{i \omega_{p}^{o}}\right)\right|\right\} \approx \frac{1}{4 \lambda \max } \operatorname{As} \operatorname{Var}\{\lambda \hat{\max }\}
$$

Next use $N r_{0} \approx\|\overline{\mathbf{u}}\|_{2}^{2}$. Hence, the asymptotic variance for the two experiment approach corresponds to

$$
\operatorname{As} \operatorname{Var}\left\{\left|\hat{G}\left(e^{i \omega_{p}^{o}}\right)\right|\right\}=\frac{\sigma_{e}^{2}(1+\delta)}{2 N r_{0}}, \quad \delta=\frac{\sigma_{e}^{2}}{2 \lambda \max ^{2} r_{0}},
$$

which should be compared to the result (25), $\sigma_{e}^{2} /\left(N r_{0}\right)$. First note that the error variance of the iterative approach decreases as $2 N$ compared with $N$ for the standard approach. The reason is that we are using twice as many measurements in the iterative approach. A fair comparison would be to use $N / 2$ data points per experiment, which would in total give $N$ measurements.

The term $\delta$ in (36) is the cost of using iterative experiment and is proportional to the noise to output ratio. This comes from the noise contribution in $y(t)$. Notice that we are only using the information in $y(t)$ from the first experiment as input to the second experiment, while still obtaining almost the same accuracy as for the direct approach.

\section{INPUT ESTIMATION}

The problem with the results given above is that we assume that the peak frequency $\omega_{p}^{o}$ and the input signal $\overline{\mathbf{u}}$ are known. For the FIR estimation we have, e.g., to apply a white input signal to estimate all impulse response coefficients. A signal with excitation of order $n+1$ or higher is needed to estimate $n+1$ parameters. A frequency domain approach would need excitation at all frequencies.

We will next summarize some of the results from [17] for estimation of the input sequence. Consider the problem of maximizing

$$
\bar{V}(\mathbf{u})=\frac{\mathbf{u}^{T} \tilde{\mathbf{z}}}{\mathbf{u}^{T} \mathbf{u}}, \quad \text { s.t. } \quad \mathbf{u}^{\mathrm{T}} \mathbf{u}=\gamma>0,
$$

with respect to $\mathbf{u}$. Recall from (28) that $\tilde{\mathbf{z}}$ is a function of u. The expected value of $\bar{V}(\mathbf{u})=\left[\mathbf{u}^{T} \mathbf{G}^{T} \mathbf{G u}+\mathbf{u}^{T} \overline{\mathbf{v}}\right] / \mathbf{u}^{T} \mathbf{u}$ is $V(\underline{\mathbf{u}})=\left[\mathbf{u}^{T} \mathbf{G}^{T} \mathbf{G u}\right] / \mathbf{u}^{T} \mathbf{u}$. Here $\overline{\mathbf{v}}=\mathbf{G}^{T} \mathbf{e}_{1}+\tilde{\mathbf{e}}_{2}$. The gradient of $V(\mathbf{u})$ equals

$$
\frac{d \bar{V}(\mathbf{u})}{d \mathbf{u}}=\frac{2}{\mathbf{u}^{T} \mathbf{u}}\left[\tilde{\mathbf{z}}-\frac{\overline{\mathbf{v}}}{2}-\left[\frac{\mathbf{u}^{T} \tilde{\mathbf{z}}}{\mathbf{u}^{T} \mathbf{u}}\right] \mathbf{u}\right]
$$

The noise term $\overline{\mathbf{v}} / 2$ is non-measurable and will be approximated by its zero mean value in the algorithms to follow. We 
then obtain the approximate (exact when there is no noise) gradient expression

$$
\nabla \bar{V}(\mathbf{u})=\frac{2}{\mathbf{u}^{T} \mathbf{u}}[\widetilde{\mathbf{z}}-\bar{V}(\mathbf{u}) \mathbf{u}]
$$

Notice that $\mathbf{u}^{T} \nabla \bar{V}(\mathbf{u})=0$, which means that the (average) gradient at $\mathbf{u}$ is orthogonal to $\mathbf{u}$. This observation makes it possible to define a gradient update that automatically satisfies the constraint $\mathbf{u}^{T} \mathbf{u}=\gamma$, namely

$$
\mathbf{u}_{k+1}=\mathbf{u}_{k} \cos \left(\theta_{k}\right)+\sqrt{\gamma} \frac{\nabla \bar{V}\left(\mathbf{u}_{k}\right)}{\left\|\nabla V\left(\mathbf{u}_{k}\right)\right\|_{2}} \sin \left(\theta_{k}\right) .
$$

If $\mathbf{u}_{k}^{T} \mathbf{u}_{k}=\gamma$ then $\mathbf{u}_{k+1}^{T} \mathbf{u}_{k+1}=\gamma$ for all $\theta_{k}$. As described in [3], this is the gradient update on a so-called Grassmann Manifold and forms the foundation of conjugate gradient algorithms. See [1] for signal processing optimization applications with unitary matrix constraint.

To our knowledge less is known about gradient based methods for calculating the maximum eigenvalue and corresponding eigenvector in an additive noise stochastic setting. Classical results in numerical analysis consider mainly roundoff errors and deterministic perturbations of matrix elements. It is known, see, e.g., [8], that the step-length of stochastic gradient methods should tend to zero as a function of the number of iterations in order to have convergence with probability one. This implies that $\theta_{k}$ in (39) should tend to zero, typically as $1 / k$, as the number of iterations $k$ increases. In practice it is often better to do a line search within a local neighborhood of $\mathbf{u}_{k}$. This can be done at the price of an extra

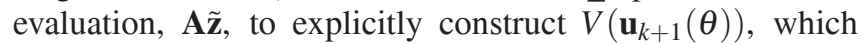
then can be maximized as a function of admissible $\theta$. This idea is closely related to Lanczos Method, and the corresponding $\theta$ optimization problem can be re-formulated as a generalized eigenvalue problem.

\section{CONCLUSIONS}

The objective of this paper has been to present and analyze some methods of gain estimation for dynamical systems. A key issue is the choice of input excitation. It is shown that the minimum variance input signal with a guaranteed accuracy is a sinusoidal signal with frequency equal to the peak frequency of the system. The corresponding frequency is in many cases unknown, but can in principle be pre-estimated using an extra initial experiment. A broad band signal such as white noise will give a variance proportional to the number of excitation frequencies or the number of parameters in the FIR model. The iterative methods extend this idea to multiple experiments and let the system generate the optimal input. We have provided new insights into the connection between these two approaches to gain estimation. There is, however, more work to be done on for example the convergence properties of iterative methods.

\section{REFERENCES}

[1] T. Abrudan, J. Eriksson, and V. Koivunen. Conjugate gradient algorithm for optimization under unitary matrix constraint. Signal Processing, 89(9):1704-1714, Sep. 2009.

[2] M-A. Massoumnia annd R.L. Kosut. A family of norms for system identification problems. IEEE Trans. on Automatic Control, 39(5):1027-1031, May 1994.

[3] A. Edelman, T.A. Arias, and S.T. Smith. The geometry of algorithms with orthogonality constraints. SIAM J. Matrix Anal. Appl, 20:303-353, 1998.

[4] G. H. Golub and C.F. van Loan. Matrix Computations. The Johns Hopkins University Press, Baltimore, 1983.

[5] H. Hjalmarsson. System identification of complex and structured systems. European Journal of Control, pages 275-231, 2009.

[6] T. Kailath. Linear Systems. Prentice-Hall, Englewood Cliffs, New Jersey, 1980.

[7] H.K. Khalil. Nonlinear Systems, third edition. Prentice Hall, Upper Saddle River, New Jersey, 2002.

[8] H. J. Kushner and G. G. Yin. Stochastic Approximation and Recursive Algorithms and Applications, second ed. Stochastic Modelling and Applied Probability, Vol. 35. Springer, 2003.

[9] L. Ljung. System Identification: Theory for the User. PrenticeHall, Englewood Cliffs, NJ, 2nd edition, 1999.

[10] L. Ljung and B. Wahlberg. Asymptotic properties of the leastsquares method for estimating transfer functions and disturbance spectra. Advances in Applied Probability, 24(2):412440, 1992.

[11] R.K. Mehra. Optimal input signals for parameter estimation in dynamic systems - Survey and new results. IEEE Transactions on Automatic Control, 19(6):753-768, 1974.

[12] J. Pintelon and J. Schoukens. System Identification: A Frequency Domain Approach. IEEE press, New York, 2001.

[13] C. R. Rojas, J. C. Agüero, J. S. Welsh, and G. C. Goodwin. On the equivalence of least costly and traditional experiment design for control. Automatica, 44(11):2706-2715, 2008.

[14] T. Söderström and P. Stoica. System Identification. PrenticeHall International, Hemel Hempstead, Hertfordshire, 1989.

[15] P. Stoica, A. Jakobsson, and J. Li. Cisoid parameter estimation in the colored noise case: asymptotic Cramer-Rao bound, maximum likelihood, and nonlinear least-squares. Signal Processing, IEEE Transactions on, 45(8):2048 -2059, Aug. 1997.

[16] P. Stoica and R. Moses. Spectral Analysis of Signals. Prentice Hall Press, Upper Saddle River, NJ, 2005.

[17] B. Wahlberg, M. Barenthin Syberg, and H. Hjalmarsson. Nonparametric methods for L2-gain estimation using iterative experiments. Automatica, 46(8):1376-1381, August 2010.

[18] B. Wahlberg, H. Hjalmarsson, and P Stoica. On optimal input signal design for frequency response estimation. In Proceedings IEEE Conference on Decision and Control, December 2010. Accepted for publication. 